\title{
Traffic-driven epidemic spreading in multiplex networks
}

\author{
Jie Chen $\odot$, Mao-Bin $\mathrm{Hu} \odot{ }^{*}$, and Ming $\mathrm{Li}^{\dagger}{ }^{\dagger}$ \\ School of Engineering Science, University of Science and Technology of China, Hefei 230026, People's Republic of China
}

(Received 31 August 2019; published 9 January 2020)

\begin{abstract}
Recent progress on multiplex networks has provided a powerful way to abstract the diverse interaction of a network system with multiple layers. In this paper, we show that a multiplex structure can greatly affect the spread of an epidemic driven by traffic dynamics. One of the interesting findings is that the multiplex structure could suppress the outbreak of an epidemic, which is different from the typical finding of spread dynamics in multiplex networks. In particular, one layer with dense connections can attract more traffic flow and eventually suppress the epidemic outbreak in other layers. Therefore, the epidemic threshold will be larger than the minimal threshold of the layers. With a mean-field approximation, we provide explicit expressions for the epidemic threshold and for the onset of suppressing epidemic spreading in multiplex networks. We also provide the probability of obtaining a multiplex configuration that suppresses the epidemic spreading when the multiplex is composed of: (i) two Erdős-Rényi layers and (ii) two scale-free layers. Therefore, compared to the situation of an isolated network in which a disease may be able to propagate, a larger epidemic threshold can be found in multiplex structures.
\end{abstract}

DOI: 10.1103/PhysRevE.101.012301

\section{INTRODUCTION}

Complex networks have become an efficient abstraction of the interactions between individuals in real complex systems. The theory of complex networks has led to many interesting results in various areas of interdisciplinary science [1]. Recently, the study of multiplex networks has become the new frontier in the area of complex networks [2]. Multiplex networks are proved to describe many real-life systems better, especially when these systems are composed of different but interconnected subnetworks [2-5]. Representative examples include the traffic systems with multimodal transportation, and the social networks where individuals can use multiple platforms. In social networks, an individual can use different platforms to communicate with different categories of friends: kinship, vicinity, membership of the same society, colleague in a company, etc. In the spreading of information, people will first select the subgroup of friends that are thought to be interested and then forward the information to the subgroup.

Multimodal traffic systems can be also abstracted as traffic dynamics on top of a multiplex structure. In the system, each geographical location has different entry points for different transportation media. Traffic dynamics are usually modeled as the flow of elements from origin to destination nodes guided by routing paths $[6,7]$. The routing strategy and network structure can greatly influence the emergence of congestion [8-13]. Many works have been devoted to extend the traffic and spreading dynamics on isolated networks to multiplex networks [14-20]. The multiplex structure has been proved to affect the transportation process, such as traffic load dis-

\footnotetext{
*humaobin@ustc.edu.cn

†minglichn@ustc.edu.cn
}

tribution, and choice of origin and destination [15,21-23]. For example, in the multimodal traffic system, each element can choose the most appropriate media to start and end its travel. If all elements adopt the shortest paths to minimize their travel distance, this "selfish behavior" will provoke an unbalance in the traffic load of the transportation layers and induce counterintuitive congestion $[15,23]$.

In this paper, we focus our study on the traffic-driven epidemic problem in multiplex networks. For convenience, we briefly review the main results of epidemic dynamics on networks. As epidemic dynamics is concerned, the structure of a network is vital to the outbreak of an epidemic [24-26]. One of the most important findings is the vanished epidemic threshold on scale-free (SF) networks [24,25,27]. The generalization of classical epidemic models [such as the susceptible-infected susceptible (SIS) and susceptibleinfected recovered] to the multiplex networks has stimulated a great interest. Obviously, one important issue is to relate the epidemic onset of the whole system with those corresponding to the isolated networks. Previous studies have pointed out that an epidemic can be induced in the multiplex system even for infection rates for which a disease would be unable to propagate in each isolated network $[19,20,28]$. That is, the critical epidemic point of the multiplex network is smaller than any of the isolated layers. The coupled system can sustain an epidemic even when the isolated networks would be free of disease.

However, in practice, the spreading of an epidemic is often accomplished with traffic dynamics, such as the outbreak of a virus in the Internet through data transmission, and the propagation of annual influenza by air travel $[29,30]$. When the epidemic spreading is taking place in a traffic-driven environment, the situation will be more complex. In fact, the outbreak of such an epidemic is mainly dependent on the spreading scope, which is determined by traffic conditions 


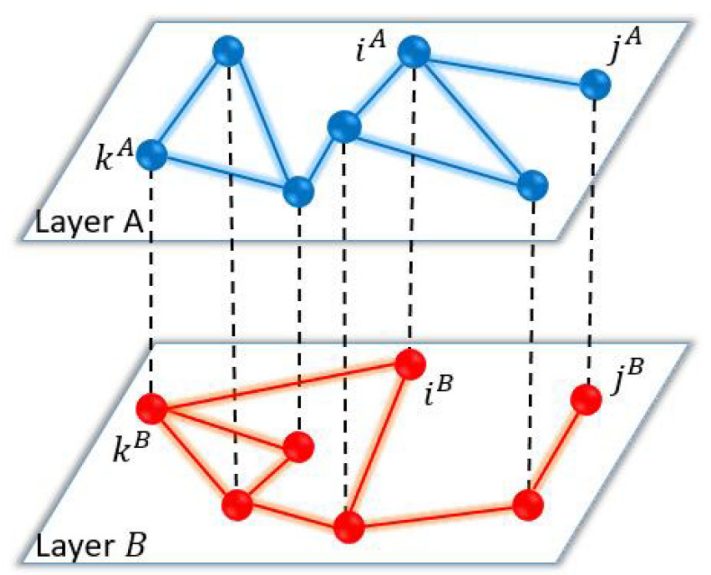

FIG. 1. Schematic of the multiplex networks. Without loss of generality, here the multiplex network is assumed to be double layered, labeled $A$ and $B$, respectively. For traffic dynamics, elements travel from the origins to the destinations following the shortest paths. For example, if an element travels from $i$ to $j$, the shortest path is $i^{A} \rightarrow j^{A}$ with length 1 . Since only layer $A$ is used, this is an intralayer path. Another example, the path length is 3 from $j$ to $k$, i.e., $j^{A} \rightarrow i^{A} \rightarrow i^{B} \rightarrow k^{B}$. Obviously, this is an interlayer path.

[27,31-35]. Due to the distribution of traffic flow among the layers, the relation of the epidemic onset of the whole system with those of the constituent layers is still unclear. In this paper, we study the traffic-driven epidemic problem with a traffic model similar to the multimodal traffic system [15]. By mean-field approximation, we obtain analytical expressions for the epidemic thresholds of the multiplex network. We show that the epidemic threshold of the multiplex network is not always smaller than those of the isolated networks. An appropriate structure of one layer will attract some traffic flow and suppress the epidemic spreading in the whole system. The suppression of an epidemic by a multiplex network will occur when the structure of constitutive layers meets some special requirements. The theoretical analysis provided in this paper can be applied to both homogeneous random multiplex networks and heterogeneous scale-free multiplex networks. The results reveal a profound relation between the outbreak of the epidemic and the multiplex structure.

\section{MODEL CONSTRUCTION}

Our model is defined on a multilayer network with $L$ layers as illustrated in Fig. 1. There are $N$ nodes in each layer. The interconnectivity between layers is assumed to be one to one, i.e., a node has only one neighbor in the other layer. It can be also understood as that a node has one replica in each layer. This network can be regarded as the excellent proxy of the structure of multimodal traffic or communication systems in geographic areas. Each location of the system has different replicas that represent each entry point to the system using the different traffic or communication media. The traffic dynamics and epidemic spreading will proceed in the system, simultaneously.

\section{A. Traffic dynamics model}

To model the traffic dynamics on a multiplex network, new transport elements are constantly generated with randomly chosen origin and destination nodes. The elements traverse the multiplex system following the shortest paths. Once arriving at the destination, the elements are removed from the system. First-in-first-out discipline is adopted in each replica.

Since nodes have replicas in each layer, an element can choose any replica of its origin node as the starting point. Arriving at any one of the replicas of the destination node is deemed to have completed the travel. Obviously, there are two types of the shortest paths for a given node pair, i.e., paths that only use a single layer (intralayer paths) and that use more than one layer (interlayer paths). Furthermore, we assume that the element generation rate of each node is $L r$, where $r$ is the average generation rate for its replica in each layer.

Due to the concern of the epidemic spreading, we will not consider the traffic congestion in this model. We assume that both the processing capacity and the buffer of each node are infinite.

\section{B. Epidemic spreading model}

Here, the classical SIS epidemic model [36] is adopted. In this model, the individuals are divided into two states, i.e., susceptible and infected. When a susceptible individual receives an element from an infected neighbor, it will be infected with probability $\beta$ or keep susceptible by the immune resources with probability $1-\beta$. At the same time, the infected individuals are recovered at rate $\mu$ (here, we set $\mu=1$ ).

Initially, all nodes in the network are susceptible, and no epidemic spreading occurs. After a transient time, the traffic dynamics reaches the steady state. Then, some replicas (a fraction $\rho_{0}$ at each layer) are randomly set as the infection sources. In all subsequent numerical experiments, $\rho_{0}=0.1$ is used. Once a replica is infected, all elements delivered from it are infected until it recovers.

\section{DETERMINATION OF THE EPIDEMIC THRESHOLD}

For an isolated uncorrelated network, the traffic-driven epidemic threshold can be obtained by the mean-field approximation [27],

$$
\beta_{c}=\frac{\langle b\rangle}{\left\langle b^{2}\right\rangle} \frac{1}{r N},
$$

where $N$ is the number of nodes, $r$ is the element generation rate for a node, $b$ is the node between-ness, and $\langle\cdot\rangle$ means the average over all nodes. This equation indicates that the epidemic threshold is constrained by the routing paths and the traffic demand. Obviously, the outbreak of an epidemic will be difficult for a light traffic load.

The situation becomes complex when the system involves more than one layer since the traffic load will redistribute over the layers. As indicated by Eq. (1), the distribution of routing paths (or the corresponding between-ness value) is the main factor that influences the spread of the epidemic. To obtain the epidemic threshold for multiplex networks, we need to first figure out the distribution of shortest paths in the system. Two important parameters have to be found: (i) $\lambda$, the fraction of 
interlayer paths among all shortest paths; (ii) $\phi^{l}$, the fraction of intralayer paths of layer $l$ in all intralayer paths. With these two parameters, we can express the between-ness of each replica approximatively as [15]

$$
b_{i}^{l} \approx \lambda \phi^{l} b_{i}^{(l)},
$$

where $b_{i}^{l}$ is the between-ness of the replica of node $i$ in layer $l$ and $b_{i}^{(l)}$ is the corresponding between-ness by treating layer $l$ as a single network. The term $\lambda \phi^{l}$ accounts for the fraction of the shortest paths traversing only layer $l$. Note that the contribution from the paths that use interlayer edges is ignored. If a particular layer has very dense connections, its original path length is relatively shorter, and there will be no need to migrate its shortest path to other layers. Especially, with the increase in average degree, one can have $\lambda \approx 1.0$ and $b_{i}^{l} \approx \phi^{l} b_{i}^{(l)}[15]$.

Then, we can find the following averages:

$$
\begin{gathered}
\left\langle b^{l}\right\rangle=\frac{\sum_{i=1}^{N} b_{i}^{l}}{N}=\lambda \phi^{l}\left\langle b^{(l)}\right\rangle, \\
\left\langle b^{l^{2}}\right\rangle=\frac{\sum_{i=1}^{N}\left(b_{i}^{l}\right)^{2}}{N}=\lambda^{2} \phi^{l^{2}}\left\langle b^{(l)^{2}}\right\rangle .
\end{gathered}
$$

With Eq. (1), we can have two ways to predict the epidemic threshold $\beta_{c}$ of the multiplex network. First, extending Eq. (1) to consider all layers in the multiplex, we have

$$
\beta_{c}=\frac{\sum_{l=1}^{L}\left\langle b^{l}\right\rangle}{\sum_{l=1}^{L}\left\langle b^{l^{2}}\right\rangle} \frac{1}{r L N} .
$$

Inserting Eqs. (3) and (4) into Eq. (5), the epidemic threshold can be predicted as

$$
\beta_{c}=\frac{\sum_{l=1}^{L} \phi^{l}\left\langle b^{(l)}\right\rangle}{\sum_{l=1}^{L} \phi^{l^{2}}\left\langle b^{(l)^{2}}\right\rangle} \frac{1}{\lambda r L N} .
$$

From another point of view, the outbreak of an epidemic in any layer can indicate the outbreak in the whole system. Thus, we can first find the epidemic threshold of each layer, and the smallest one can be regarded as the epidemic threshold for the multiplex network. Following the mean-field approximation [27], the epidemic threshold for a layer should be as follows:

$$
\begin{aligned}
\beta_{c}^{l} & =\frac{\left\langle b^{l}\right\rangle}{\left\langle b^{l^{2}}\right\rangle} \frac{1}{r L N} \\
& =\frac{\left\langle b^{(l)}\right\rangle}{\phi^{l}\left\langle b^{(l)^{2}}\right\rangle} \frac{1}{\lambda r L N} .
\end{aligned}
$$

Then, the epidemic threshold of the multiplex network is

$$
\beta_{c}=\min _{l \in L}\left\{\beta_{c}^{l}\right\} .
$$

To verify and compare the accuracy of Eqs. (6) and (8), we perform a simulation experiment on double-layered ErdősRényi multiplex networks with $N=500$. The results are shown in Fig. 2. It can be seen that Eq. (8) is more accurate to predict the epidemic threshold of multiplex networks. The

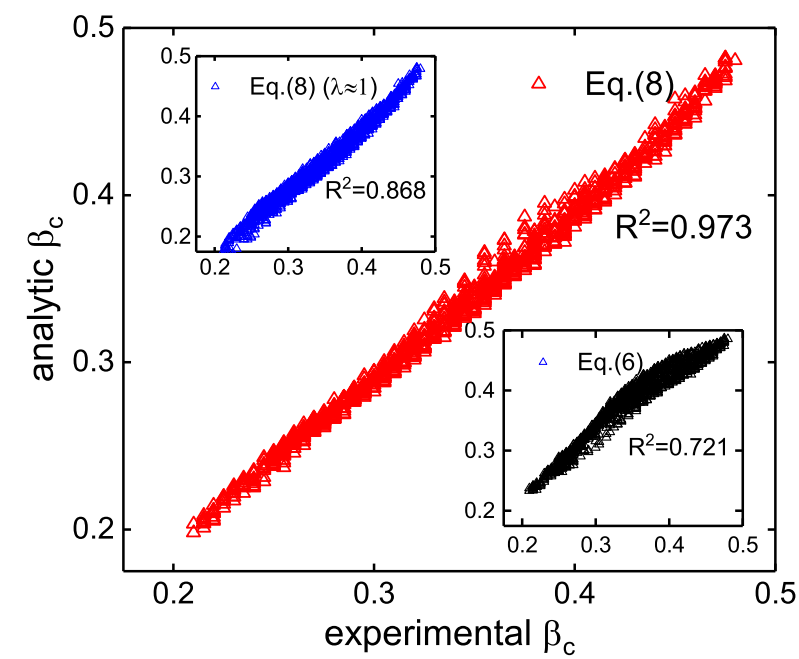

FIG. 2. Comparison of the threshold $\beta_{c}$ obtained by theoretical analysis and by experimental simulation. The multiplex networks used in the simulation are formed by two Erdős-Rényi networks ( $L=$ 2) with $N=500$. For the traffic dynamics, the element generation rate is set as $r=1$. The results are obtained by Eq. (8) (main figure), Eq. (6) (lower right), and Eq. (8) with $\lambda \approx 1$ (upper left), respectively. $R^{2}$ is the coefficient of determination for the linear fitting. Equation (8) shows the best prediction with $R^{2}=0.973$.

inferior accuracy of Eq. (6) can be explained as follows. In fact, the extension of Eq. (6) is based on the assumption of an uncorrelated network. For multiplex networks, the interconnections among different layers will make the system more correlated and degenerate the accuracy of Eq. (6).

\section{EPIDEMIC SUPPRESSED ON THE MULTIPLEX NETWORKS}

The multiplex structure can make the traffic flow immigrate from the less efficient layers (with longer travel lengths) to the more efficient layers (with shorter travel lengths) [15]. In the context of traffic-driven epidemic spreading, this redistribution of traffic flow will affect the spreading in the layers and can eventually induce or suppress the epidemic spreading. In the following, we will explore this effects in detail using both homogeneous Erdős-Rényi multiplex networks and heterogeneous scale-free multiplex networks.

\section{A. Epidemic suppressed on Erdôs-Rényi multiplex networks}

We can investigate the phenomenon of an epidemic suppressed by a multiplex structure as the situation that the epidemic threshold of the multiplex network is larger than, at least, one threshold of its layers when operating individually.

As an example, consider a multiplex structure composed of two layers $A$ and $B$ with average degrees $\left\langle k^{(A)}\right\rangle$ and $\left\langle k^{(B)}\right\rangle$. For two layers with the same number of nodes, the layer with more links (or a larger average degree) usually has a smaller average between-ness. From Eq. (1), one can find that the layer will have a larger epidemic threshold. Without loss of generality, we assume that layer $B$ is more efficient in terms of traffic dynamics (i.e., $\left\langle k^{A}\right\rangle<\left\langle k^{B}\right\rangle$, and $\beta_{c}^{(A)}<\beta_{c}^{(B)}$ ). When forming a multiplex network, since layer $B$ is more 


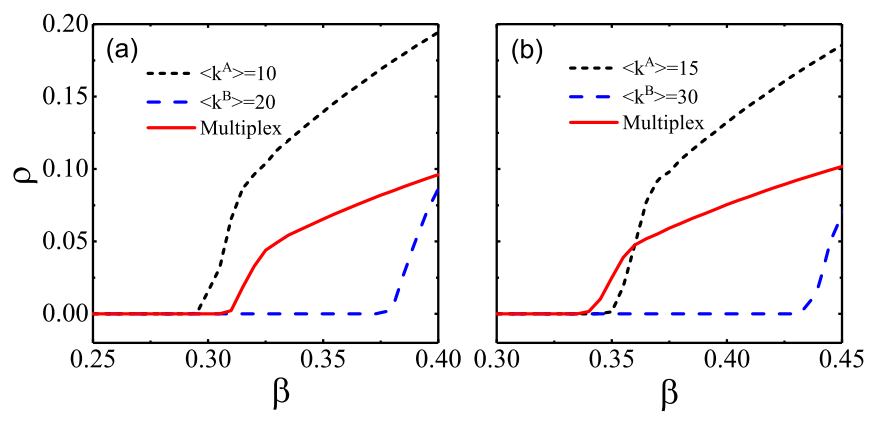

FIG. 3. Fraction of infected nodes $\rho$ as a function of infected probability $\beta$ for two typical Erdős-Rényi multiplex networks formed with two layers of different average degrees: (a) $\left\langle k^{A}\right\rangle=10$ and $\left\langle k^{B}\right\rangle=20$; (b) $\left\langle k^{A}\right\rangle=15$ and $\left\langle k^{B}\right\rangle=30$. Other parameters are $N=$ 500 and $r=1$.

efficient, the traffic flow will immigrate from layer $A$ to layer $B$. Therefore, the epidemic threshold of layer $A$ will increase, whereas the threshold of layer $B$ will decrease. That is as follows:

$$
\begin{aligned}
& \beta_{c}^{A} \gtrsim \beta_{c}^{(A)}, \\
& \beta_{c}^{B} \lesssim \beta_{c}^{(B)} .
\end{aligned}
$$

As Eq. (8) predicts, the epidemic threshold of multiplex $\beta_{c}$ will be the smaller one of $\beta_{c}^{A}$ and $\beta_{c}^{B}$,

$$
\beta_{c}=\min \left(\beta_{c}^{A}, \beta_{c}^{B}\right) .
$$

For the situation of an epidemic suppressed by the multiplex configuration, the epidemic threshold of the multiplex network should satisfy

$$
\beta_{c}>\beta_{c}^{(A)}
$$

For this situation, since $\beta_{c}^{A}$ will be always greater than $\beta_{c}^{(A)}$ [Eq. (9)], the epidemic threshold of the multiplex network should be $\beta_{c}=\beta_{c}^{B}$. Thus, we can find the condition for the occurrence of an epidemic suppressed by the multiplex configuration as: $\beta_{c}^{B}>\beta_{c}^{(A)}$. Using Eqs. (1) and (6) to determine $\beta_{c}^{(A)}$ and $\beta_{c}^{B}$, respectively, the condition can be rewritten as

$$
\frac{1}{2 \lambda \phi^{B}} \gtrsim \frac{\left\langle b^{(A)}\right\rangle\left\langle b^{(B)^{2}}\right\rangle}{\left\langle b^{(B)}\right\rangle\left\langle b^{(A)^{2}}\right\rangle} .
$$

From this inequation, we will find that a double-layered multiplex structure can produce a larger epidemic threshold than, at least, one individual layer when the condition is meet. This is verified by simulation experiments of multiplex networks composed of two Erdős-Rényi layers. Figure 3 shows the fraction of infected nodes $\rho$ as a function of infected probability $\beta$ for two typical Erdős-Rényi multiplex networks. Figure 3(a) shows a special result that the multiplex shows an epidemic threshold between the thresholds of its single layers, whereas Fig. 3(b) shows a normal result of epidemic induced by the multiplex structure.

Figure 4 shows the probability of observing the epidemic suppressed by the multiplex structure generated with ErdősRényi networks. For each data point, we examine 100 realizations of the multiplex network. For each realization, we compute the epidemic thresholds of the multiplex network

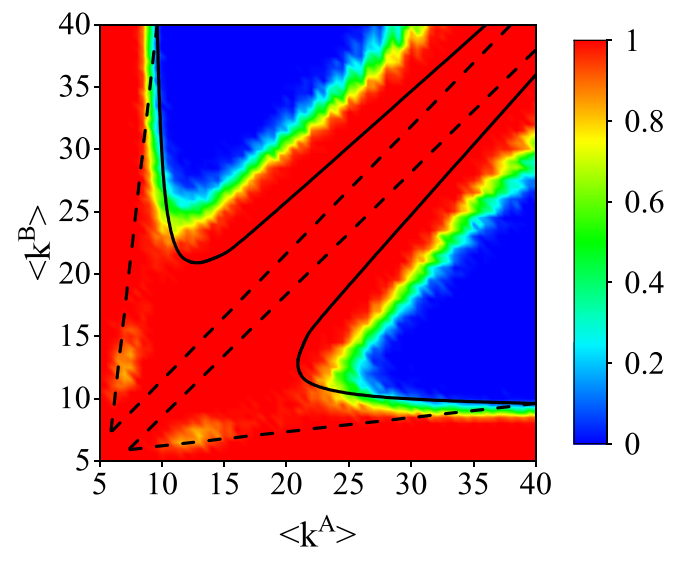

FIG. 4. Probability of obtaining a multiplex configuration that suppresses epidemic spreading. The number of points is $70^{2}$, and for each point, 100 configurations are generated with fixed $\left\langle k^{A}\right\rangle$ and $\left\langle k^{B}\right\rangle$. The colors indicate the probability of $\beta_{c}>\min \left(\beta_{c}^{(A)}, \beta_{c}^{(B)}\right)$. The lines show the result of Eq. (13) to detect the boundaries [the solid lines are obtained by Eq. (13) with the real value of $\lambda$, and the dashed lines are obtained with $\lambda=1$ ]. Other parameters are $L=2, N=500$, and $r=1$.

and of the individual layers. Then, we obtained the fraction of realization that the multiplex network has a larger epidemic threshold than one layer. The result of Eq. (13) is used to determine the regions where the multiplex suppresses the epidemic. One can see that the result of Eq. (13) shows the qualitative agreement with the experiments. The deviation is mainly caused by the mean-field approximation and the correlation of networks, especially when the average degrees of individual layers are large.

The reason behind this phenomenon is the distribution of traffic flow between the layers. The more efficient layer (in terms of the traffic flow) will attract some traffic flow from the less efficient layer, which is also more vulnerable in the epidemic spreading. When the structures of two layers are appropriate, this effect will eventually increase the epidemic threshold. However, for the region of comparatively different average degrees (upper and right edge), the probability of observing an epidemic suppressed by multiplex structure is very low. The reason of this phenomenon is also the redistribution of traffic flow among the layers. When one layer is far more efficient than the other layers, the traffic flow will immigrate to the layer, and eventually induce an epidemic outbreak in the layer.

\section{B. Epidemic suppressed on scale-free multiplex networks}

In the previous subsection, we show that the spreading of a traffic-driven epidemic may be suppressed by a multiplex network composed of homogenous random graph layers. In fact, this phenomenon can be observed in other multiplex network structures. Here, we demonstrate the suppression of a traffic-driven epidemic on SF multiplex networks, which is more appropriate to represent real-life communication systems. We construct each layer by the uncorrelated configuration model (UCM) [37,38]. The rules of the UCM proceed as follows: (1) In a set of $N$ initially disconnected nodes, assign each node $i$ a degree $k_{i}$ sampled from the power-law 


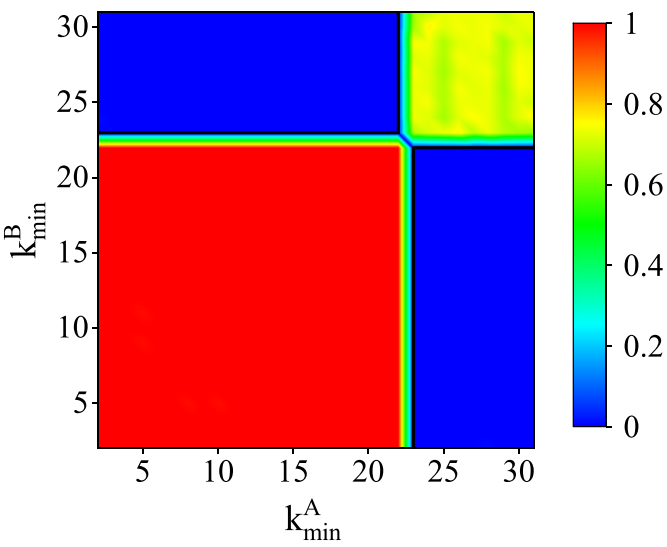

FIG. 5. Probability of obtaining a scale-free multiplex configuration that suppresses epidemic spreading. The number of simulation points is $30^{2}$, and, for each point, we generate 100 configurations fixing $k_{\min }^{A}$ and $k_{\min }^{B}$. The colors indicate the probability of observing that the epidemic threshold of the multiplex satisfies $\beta_{c}>$ $\min \left(\beta_{c}^{(A)}, \beta_{c}^{(B)}\right)$. The lines show the results of Eq. (13) in detecting the region where the multiplex structure induces an epidemic.

distribution $P(k) \sim k^{-\gamma}$ with the constraints $k_{\min } \leqslant k_{i} \leqslant k_{\max }$ (normally $k_{\max }=\sqrt{N}$ ); (2) randomly connect nodes by the preassigned degrees, and multiple and self-connections are forbidden. One can see that the parameters controlling the structure of the UCM SF networks are $k_{\min }$ and $\gamma$. Here, we fix $\gamma=2.8, N=1000$, and adjust $k_{\min }$ as a variable to control the layer structure. Furthermore, since the system used here is relatively small, the hubs could dominate the structure of the network as well as the distribution of the traffic load. So, we add a constraint $k_{\max }=\min \left(k_{\min }+10, \sqrt{N}\right)$ to distinguish layers with different $k_{\text {min }}$ 's. With the increase in the system size, this constraint can be relaxed. However, that might be computationally expensive.

Figure 5 shows the probability of observing an epidemic suppressed by the SF multiplex networks. The phenomenon of suppressing the epidemic in the multiplex networks is observed when both layers' minimal degrees are relatively small. When the minimal degree of any layer exceeds $k_{\text {min }} \approx 22$, the UCM model will make a node in the layer have the maximum degree of $k_{\max }=\sqrt{N}$. Then, the traffic flow will be attracted to the layer, and the effect will eventually produce a lower epidemic threshold for the multiplex network. Equation (13) can work well in predicting the boundaries of regions. With SF networks, the approximation of $\lambda \approx 1.0$ is no longer valid. Therefore, the prediction of Eq. (13) with $\lambda=1.0$ is not presented.

\section{CONCLUSION}

To summarize, we have studied the traffic-driven epidemic spreading phenomena on multiplex networks. We integrate the susceptible-infected-susceptible model with a traffic model and develop a theoretical method to determine the epidemic threshold of multiplex networks. By theoretical analysis, we show that the epidemic can be suppressed by the multiplex structure, which is different from previous findings that the multiplex network will always induce an epidemic. We also provide an explicit expression for determining the network parameters that trigger these phenomena. The theoretical predictions show a qualitative agreement with the empirical experiments.

The suppression of the epidemic can be observed on both Erdős-Rényi multiplex networks and scale-free multiplex networks. The reason is the redistribution of the traffic flow among the layers. When some layers are coupled to be a multiplex network, the traffic flow could immigrate from one layer to some others. Eventually, this redistribution of the traffic flow could suppress the spreading of the epidemic in the whole system. However, if one layer attracted too much traffic flow, a lower spreading threshold can also be found.

These findings reinforce the importance of multiplex structures on network dynamics. The results might also help the development of more efficient immunization policies.

\section{ACKNOWLEDGMENTS}

The research was supported by the National Natural Science Foundation of China (Grants No. 11672289 and No. 61773148).
[1] S. Boccaletti, V. Latora, Y. Moreno, M. Chavez, and D.-U. Hwang, Complex networks: Structure and dynamics, Phys. Rep. 424, 175 (2006).

[2] S. Boccaletti, G. Bianconi, R. Criado, C. I. del Genio, J. GómezGardeñes, M. Romance, I. Sendiña-Nadal, Z. Wang, and M. Zanin, The structure and dynamics of multilayer networks, Phys. Rep. 544, 1 (2014), the structure and dynamics of multilayer networks.

[3] M. De Domenico, A. Solé-Ribalta, E. Cozzo, M. Kivelä, Y. Moreno, M. A. Porter, S. Gómez, and A. Arenas, Mathematical Formulation of Multilayer Networks, Phys. Rev. X 3, 041022 (2013).

[4] P. J. Mucha, T. Richardson, K. Macon, M. A. Porter, and J.-P. Onnela, Community structure in time-dependent, multiscale, and multiplex networks, Science 328, 876 (2010).
[5] M. Kivelä, A. Arenas, M. Barthelemy, J. P. Gleeson, Y. Moreno, and M. A. Porter, Multilayer networks, J. Complex Networks 2, 203 (2014).

[6] M. Boguñá, D. Krioukov, and K. C. Claffy, Navigability of complex networks, Nat. Phys. 5, 74 (2008).

[7] D. Helbing, Traffic and related self-driven many-particle systems, Rev. Mod. Phys. 73, 1067 (2001).

[8] W.-X. Wang, B.-H. Wang, C.-Y. Yin, Y.-B. Xie, and T. Zhou, Traffic dynamics based on local routing protocol on a scale-free network, Phys. Rev. E 73, 026111 (2006).

[9] D. De Martino, L. Dall'Asta, G. Bianconi, and M. Marsili, Congestion phenomena on complex networks, Phys. Rev. E 79, 015101(R) (2009).

[10] G. Yan, T. Zhou, B. Hu, Z.-Q. Fu, and B.-H. Wang, Efficient routing on complex networks, Phys. Rev. E 73, 046108 (2006). 
[11] W.-X. Wang, C.-Y. Yin, G. Yan, and B.-H. Wang, Integrating local static and dynamic information for routing traffic, Phys. Rev. E 74, 016101 (2006).

[12] X. Ling, M.-B. Hu, R. Jiang, and Q.-S. Wu, Global dynamic routing for scale-free networks, Phys. Rev. E 81, 016113 (2010).

[13] Z. Liu, M.-B. Hu, R. Jiang, W.-X. Wang, and Q.-S. Wu, Method to enhance traffic capacity for scale-free networks, Phys. Rev. E 76, 037101 (2007).

[14] R. G. Morris and M. Barthelemy, Transport on Coupled Spatial Networks, Phys. Rev. Lett. 109, 128703 (2012).

[15] A. Solé-Ribalta, S. Gómez, and A. Arenas, Congestion Induced by the Structure of Multiplex Networks, Phys. Rev. Lett. 116, 108701 (2016).

[16] S. Manfredi, E. Di Tucci, and V. Latora, Mobility and Congestion in Dynamical Multilayer Networks with Finite Storage Capacity, Phys. Rev. Lett. 120, 068301 (2018).

[17] C. Granell, S. Gómez, and A. Arenas, Dynamical Interplay between Awareness and Epidemic Spreading in Multiplex Networks, Phys. Rev. Lett. 111, 128701 (2013).

[18] S. Gómez, A. Díaz-Guilera, J. Gómez-Gardeñes, C. J. PérezVicente, Y. Moreno, and A. Arenas, Diffusion Dynamics on Multiplex Networks, Phys. Rev. Lett. 110, 028701 (2013).

[19] H. Wang, Q. Li, G. D’Agostino, S. Havlin, H. E. Stanley, and P. Van Mieghem, Effect of the interconnected network structure on the epidemic threshold, Phys. Rev. E 88, 022801 (2013).

[20] A. Saumell-Mendiola, M. A. Serrano, and M. Boguñá, Epidemic spreading on interconnected networks, Phys. Rev. E 86, 026106 (2012).

[21] F. Tan, J. Wu, Y. Xia, and C. K. Tse, Traffic congestion in interconnected complex networks, Phys. Rev. E 89, 062813 (2014).

[22] M. Li, M.-B. Hu, and B.-H. Wang, Transportation dynamics on coupled networks with limited bandwidth, Sci. Rep. 6, 39175 (2016).

[23] A. Solé-Ribalta, A. Arenas, and S. Gómez, Effect of shortest path multiplicity on congestion of multiplex networks, New J. Phys. 21, 035003 (2019).

[24] C. Castellano and R. Pastor-Satorras, Thresholds for Epidemic Spreading in Networks, Phys. Rev. Lett. 105, 218701 (2010).
[25] R. Pastor-Satorras and A. Vespignani, Epidemic Spreading in Scale-Free Networks, Phys. Rev. Lett. 86, 3200 (2001).

[26] R. Parshani, S. Carmi, and S. Havlin, Epidemic Threshold for the Susceptible-Infectious-Susceptible Model on Random Networks, Phys. Rev. Lett. 104, 258701 (2010).

[27] S. Meloni, A. Arenas, and Y. Moreno, Traffic-driven epidemic spreading in finite-size scale-free networks, Proc. Natl. Acad. Sci. U.S.A. 106, 16897 (2009).

[28] E. Cozzo, R. A. Baños, S. Meloni, and Y. Moreno, Contactbased social contagion in multiplex networks, Phys. Rev. E 88, 050801(R) (2013).

[29] C. Viboud, O. N. Bjørnstad, D. L. Smith, L. Simonsen, M. A. Miller, and B. T. Grenfell, Synchrony, waves, and spatial hierarchies in the spread of influenza, Science 312, 447 (2006).

[30] X. Han and Q. Tan, Dynamical behavior of computer virus on internet, Appl. Math. Comput. 217, 2520 (2010).

[31] H.-X. Yang, W.-X. Wang, Y.-C. Lai, Y.-B. Xie, and B.-H. Wang, Control of epidemic spreading on complex networks by local traffic dynamics, Phys. Rev. E 84, 045101(R) (2011).

[32] H.-X. Yang, Z.-X. Wu, and B.-H. Wang, Suppressing trafficdriven epidemic spreading by edge-removal strategies, Phys. Rev. E 87, 064801 (2013).

[33] H.-X. Yang, M. Tang, and Y.-C. Lai, Traffic-driven epidemic spreading in correlated networks, Phys. Rev. E 91, 062817 (2015).

[34] L. Gao, P. Shu, M. Tang, W. Wang, and H. Gao, Effective trafficflow assignment strategy on multilayer networks, Phys. Rev. E 100, 012310 (2019).

[35] M. Li, R.-R. Liu, D. Peng, C.-X. Jia, and B.-H. Wang, Roles of the spreading scope and effectiveness in spreading dynamics on multiplex networks, Physica A 492, 1239 (2018).

[36] N. T. J. Bailey et al., The Mathematical Theory of Infectious Diseases and Its Applications (Charles Griffin, London, 1975).

[37] M. Catanzaro, M. Boguñá, and R. Pastor-Satorras, Generation of uncorrelated random scale-free networks, Phys. Rev. E 71, 027103 (2005).

[38] S. N. Dorogovtsev, A. V. Goltsev, and J. F. F. Mendes, Critical phenomena in complex networks, Rev. Mod. Phys. 80, 1275 (2008). 